\title{
A Novel Genetic Variant in the WFS1 Gene in a Patient with Partial Uniparental Mero-Isodisomy of Chromosome 4
}

\author{
Maurizio Delvecchio ${ }^{1, *}\left(\mathbb{D}\right.$, Federica Ortolani $^{1}$, Orazio Palumbo ${ }^{2}{ }^{2}$, Concetta Aloi $^{3}$, Alessandro Salina ${ }^{3}$, \\ Francesco Claudio Susca ${ }^{4}$, Pietro Palumbo ${ }^{2} \mathbb{D}$, Massimo Carella ${ }^{2} \mathbb{D}$, Nicoletta Resta ${ }^{4}(\mathbb{D})$ and Elvira Piccinno ${ }^{1}$ \\ 1 Metabolic Disease and Genetics Disorders Unit, Giovanni XXIII Children's Hospital, 70126 Bari, Italy; \\ federicaortolani@hotmail.com (F.O.); lorenzoeluigi@libero.it (E.P.) \\ 2 Medical Genetics Unit, IRCCS Casa Sollievo della Sofferenza, 71013 San Giovanni Rotondo (FG), Italy; \\ o.palumbo@operapadrepio.it (O.P.); p.palumbo@operapadrepio.it (P.P.); m.carella@operapadrepio.it (M.C.) \\ 3 Clinica Pediatrica, LABSIEM, IRCCS Istituto Giannina Gaslini, 16147 Genoa, Italy; \\ concettaaloi@gaslini.org (C.A.); alessandrosalina@gaslini.org (A.S.) \\ 4 Department of Biomedical Sciences and Human Oncology (DIMO), Division of Medical Genetics, University \\ of Bari “Aldo Moro", 70125 Bari, Italy; francescoclaudio.susca@uniba.it (F.C.S.); nicoletta.resta@uniba.it (N.R.) \\ * Correspondence: mdelvecchio75@gmail.com; Tel.: +39-08-0559-6771
}

\section{check for} updates

Citation: Delvecchio, M.; Ortolani, F.; Palumbo, O.; Aloi, C.; Salina, A.; Susca, F.C.; Palumbo, P.; Carella, M.; Resta, N.; Piccinno, E. A Novel Genetic Variant in the WFS1 Gene in a Patient with Partial Uniparental Mero-Isodisomy of Chromosome 4. Int. J. Mol. Sci. 2021, 22, 8082 https://doi.org/10.3390/ ijms22158082

Academic Editor: Gil Atzmon

Received: 25 June 2021

Accepted: 25 July 2021

Published: 28 July 2021

Publisher's Note: MDPI stays neutra with regard to jurisdictional claims in published maps and institutional affiliations.

Copyright: (c) 2021 by the authors. Licensee MDPI, Basel, Switzerland. This article is an open access article distributed under the terms and conditions of the Creative Commons Attribution (CC BY) license (https:// creativecommons.org/licenses/by/ $4.0 /)$.

\begin{abstract}
Wolfram syndrome is a rare autosomal recessive disorder characterized by optic atrophy and diabetes mellitus. Wolfram syndrome type 1 (WFS1) is caused by bi-allelic pathogenic variations in the wolframin gene. We described the first case of WFS1 due to a maternal inherited mutation with uniparental mero-isodisomy of chromosome 4 . Diabetes mellitus was diagnosed at 11 years of age, with negative anti-beta cells antibodies. Blood glucose control was optimal with low insulin requirement. No pathogenic variations in the most frequent gene causative of maturity-onset diabetes of the young subtypes were detected. At 17.8 years old, a rapid reduction in visual acuity occurred. Genetic testing revealed the novel homozygous variant c.1369A>G; p.Arg457Gly in the exon 8 of wolframin gene. It was detected in a heterozygous state only in the mother while the father showed a wild type sequence. In silico disease causing predictions performed by Polyphen 2 classified it as "likely damaging", while Mutation Tester and Sift suggested it was "polymorphism" and "tolerated", respectively. High resolution SNP-array analysis was suggestive of segmental uniparental disomy on chromosome 4 . In conclusion, to the best of our knowledge, we describe the first patient with partial uniparental mero-isodisomy of chromosome 4 carrying a novel mutation in the wolframin gene. The clinical phenotype observed in the patient and the analysis performed suggest that the genetic variant detected is pathogenetic.
\end{abstract}

Keywords: Wolfram syndrome; uniparental disomy; chrosome 4; wolframin; syndromic diabetes

\section{Introduction}

Wolfram syndrome type 1 (WFS1; OMIM \#222300) is a rare autosomal recessive disorder whose minimal diagnostic criteria are optic atrophy $(\mathrm{OA})$ and juvenile onset non-autoimmune diabetes mellitus (DM). These features may be present in childhood but also later in life, and typically, but not invariably, diabetes mellitus is detected first. It is also known with the acronym of DIDMOAD syndrome as the other pivotal clinical features are usually diabetes insipidus (DI) and progressive sensorineural deafness (D). In addition, urinary tract abnormalities, ataxia, dementia or intellectual disability, and psychiatric illnesses could be observed [1]. From a molecular point of view, Wolfram syndrome is caused by loss of function homozygous or compound heterozygous pathogenic variations in the gene WFS1. The gene is mapped to chromosome region 4p16 and encodes for wolframin, a transmembrane protein, which plays a pivotal role in membrane trafficking, secretion, processing, and/or regulation of endoplasmic reticulum (ER) calcium homeostasis [2]. 
To date and to the best of our knowledge, over 170 different pathogenic variations have been reported and linked to the disease [3]. Missense changes that lead to amino acid substitution represent the most frequent molecular consequence, while nonsense and frameshift are less frequent. Most of the variants are located on exon 8. The clinical course is highly variable, even within the same family, and a genotype-phenotype correlation is a difficult task due to the molecular complexity of Wolfram syndrome and the different clinical characteristics $[4,5]$.

In this manuscript, we report on the first case of WFS1 due to a maternally inherited mutation with partial uniparental mero-isodisomy of chromosome 4.

\section{Results}

Genetic testing revealed the novel homozygous pathogenic variant c.1369A>G; p.Arg457Gly in exon 8 of WFS1 gene (NM_006005.3) (Figure 1). It was found in a heterozygous state in the mother, but it was not detected in the father and has not yet been reported in the literature or in the Exac database. The sibling showed a wild type sequence. In silico analysis by Polyphen 2 indicated this variant as "likely damaging", while Mutation Tester and Sift classified it as "polymorphism" and "tolerated", respectively. Following this pipeline, no clinically significant copy number changes were identified, while two wide regions of homozygosity that were suggestive of partial uniparental mero-isodisomy disomy (UPD) have been identified on chromosome 4 (UPD4) (Figure 2).

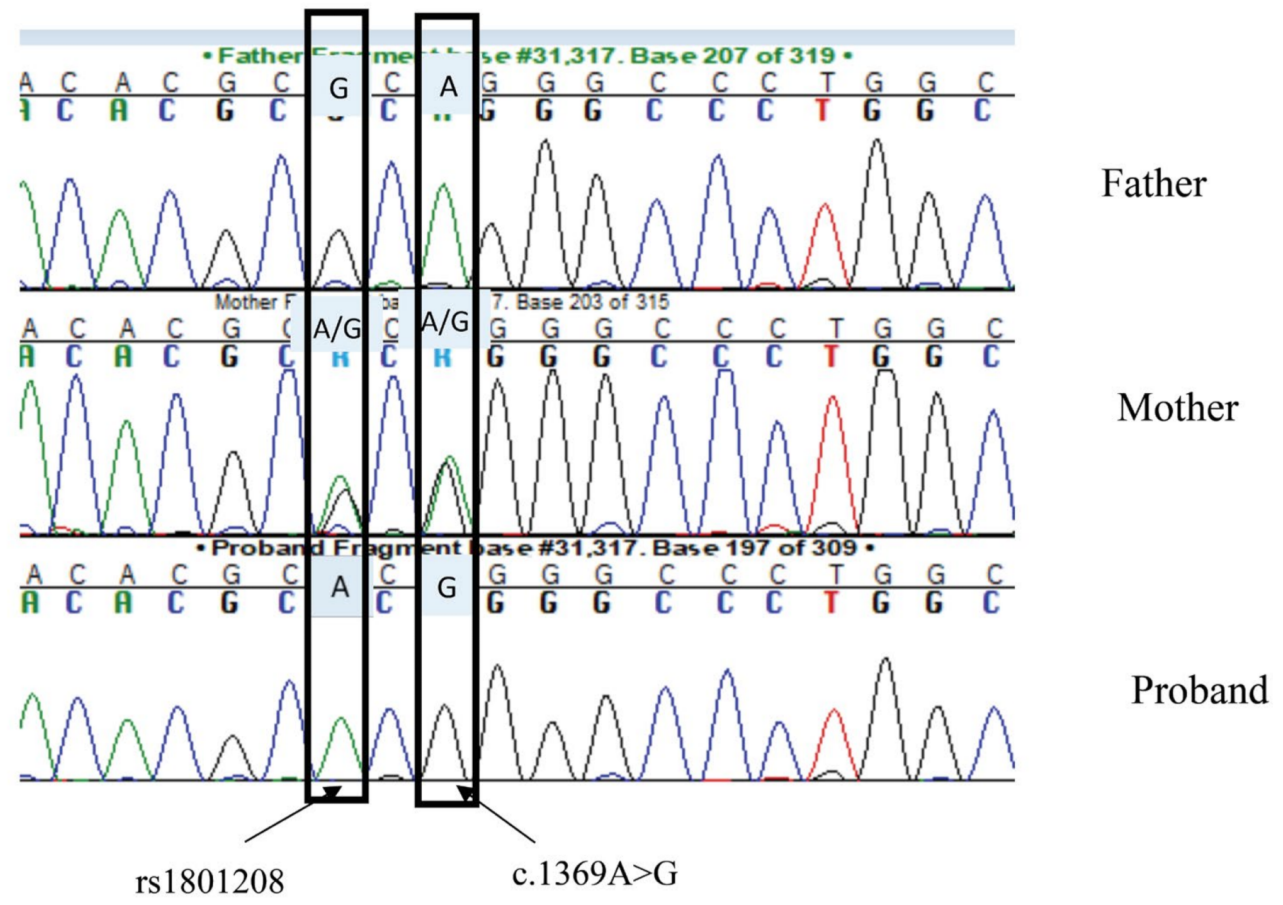

Figure 1. Segregation of WFS1 novel missense variant c.1369A>G; p.Arg457Gly and rs1801208 in family pedigree. Electopherogram shows that proband was the missense variant and SNP were detected in a homozygous state in the proband and in a heterozygous state in the mother. The father was wild type for both alleles. 


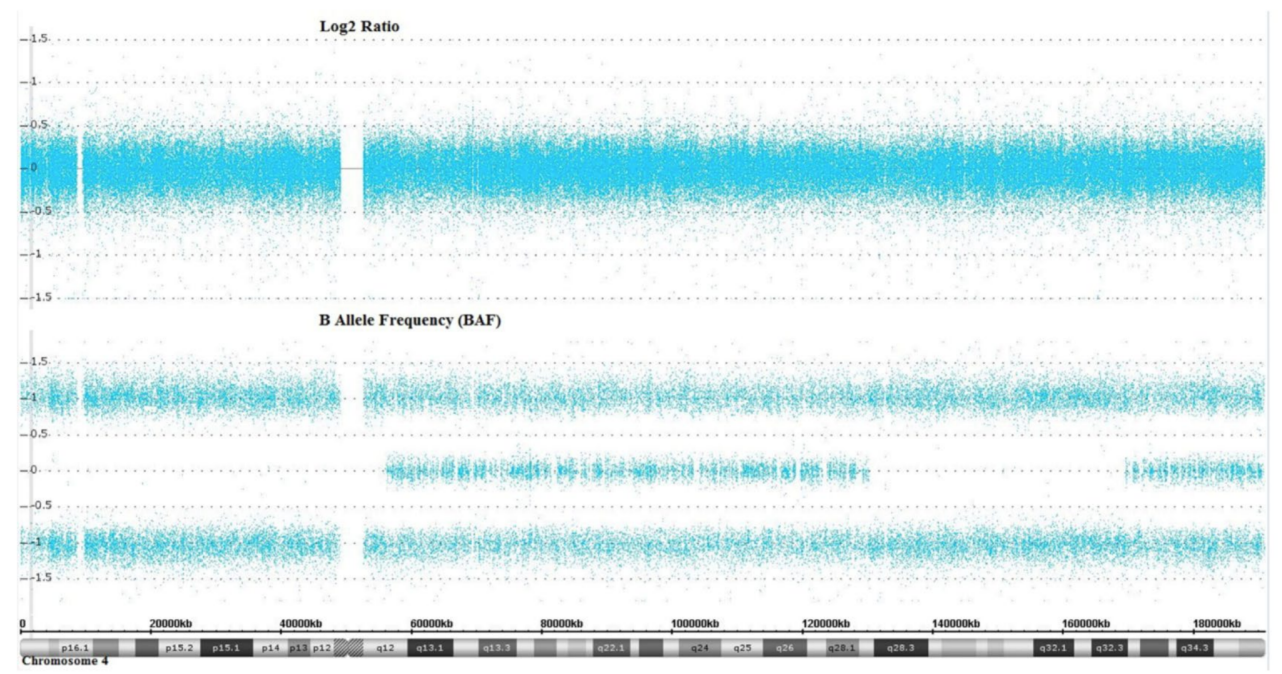

Figure 2. SNP-arrays identified the presence of a segmental UPD on chromosome 4 . The log2 ratio indicates a normalized intensity value of each SNP across chromosome 4 compared to diploid individuals. In detail, the $\log 2$ ratio of 0 indicates the absence of copy number alterations (i.e., microdeletions and/or microduplications) on chromosome 4 in our patient compared to controls. The B allele can have values of $1(\mathrm{BB}), 0(\mathrm{AB})$ and $-1(\mathrm{AA})$ in a diploid individual. Data show that the patient had a segmental UPD spanning the chromosome $4 p$ and $4 q$ regions. In detail, the $B$ allele frequency shows a 56.1-Mb loss of heterozygosity at chromosome 4p16.3q12 (46,690-56,195,626; hg19), and a 39.4-Mb loss of heterozygosity at chromosome 4q28.2q32.3 (130,191,096-169,630,204; hg19). Genotype analysis confirmed an allelic imbalance compatible with maternal chromosome 4p16.3q12 and 4q28.2q32.3 UPD (data not shown).

\section{Discussion}

The clinical diagnosis of Wolfram syndrome is based on the association of DM and OA, while the genetic diagnosis relays on the detection of pathogenic variants in both of the WFS1 gene alleles. We have previously described a very rare case of Wolfram syndrome type 2 [6] and here we report the first case of WFS1 caused by the c.1369A>G; p.Arg457Gly homozygous variant of the WFS1 gene as a consequence of partial mero-isodisomy of maternal chromosome 4 . This novel missense change was found in a homozygous state in the proband and it was absent from databases such as gnomAD, 1000 Genomes and dbSNP. In 2005, Giuliano et al., described in the same position, 457, the substitution of Arginine 457 with another amminoacid the Serine detected in heterozigousity in a WFS1 patient with an atypical phenotype [7]. In our case, the segregation of the genetic variant with the typical phenotype leads us to consider this genetic variant as "likely pathogenic". The unusual maternal inheritance is related to the partial maternal UPD of chromosome 4 . This event is rare but not impossible, causing homozigosity for a genetic variant carried by just one of the parents, and thus confirming the diagnosis of Wolfram syndrome.

Mero-isodisomy may coexist on the same pair of uniparental chromosomes due to one or more recombination events in the prophase of the first meiotic division; afterward, meiotic chromosome segregation errors (non-disjunction at meiosis I or meiosis II, but also reverse segregation and premature separation of chromatids) originate an disomic/nullisomic gamete. The mitotic correction of the resulting aneuploid embryo (trisomy/monosomy rescue) may cause UPD. The UPD phenotypic effects may be due to the involvement of imprinting regions or isodisomy, and can result in a recessive disorder caused by a homozygous pathogenic variant that is present in only one of the parents $[8,9]$.

The SNP array analysis of our patient suggests an MII segregation error of chromosome 4 in maternal meiosis, prior to the meiotic segregation three cross-over events having taken place between the long arms of the homologous chromosomes, then recombinant and non-recombinant sister chromatids are segregated into a disomic egg. A trisomic 
conceptus is produced following fertilization. The rescue of the trisomy by another error in postzygotic mitosis (paternal chromosome 4 was discarded) results in maternal UPD4 with isodisomy for non-recombinat regions (4p16.3 proximal and 4q28.2q32.3 interstitial) and heterodisomy for recombinant regions (4q12q28.1 interstitial and 4q32.3qter distal). WFS1 gene variant maps to $4 \mathrm{p} 16$.

A few dozen cases of isodisomy unmasking a mutant recessive allele have been reported, even few cases of UPD4 [10-14]. In most cases, the sequence of the events was similar to that described in our case, but also one case of paternal UPD4 [15] and two cases of incidental findings $[16,17]$ have been described.

The incidence of UPD is mainly ascertained through an abnormal phenotype due to an imprinting disorder or recessive disorder, but a recent study on the frequency of UPD in a large population of mostly healthy individuals estimates that the frequency of whole chromosome UPD is approximately 1 in 2000 live births [18]. Maternal UPD is more frequent than paternal UPD and increases with maternal age; however low the risk of UPD, it is necessary to take this into account for genetic counselling as a cause of autosomal recessive disorders, especially when a rare homozygous pathogenic variant is detected.

A few years ago, Papadimitriou et al. [19] described an unusual WFS1 presentation in a patient with maternal UPD4 with a homozygous mutation in the wolframin gene. The proband was a 10-year-old girl with type $1 \mathrm{DM}$ diagnosed at 6 years of age, high-frequency sensorineural hearing loss, and a reduction in visual acuity. Her parents and her brother were all healthy. The father and the brother did not carry the defect, which was found in her mother in a heterozygous state. A SNP array analysis for genotyping and a microsatellite analysis to determine the origin of the second allele were performed, showing UPD due to uniparental segregation of the maternal chromosomes. However, our patient carries a mero-isodisomy of maternal chromosome 4, while the patient described by Papadimitrou et al., presented with UPD.

Finally, we would like to report on the parents' response. We discussed the diagnosis of WFS1 after the genetic testing with them. We asked about their feeling in the case of diagnosis when the patient was 12 years old. They replied that they were happy that the diagnosis had not been conducted before, when she presented only diabetes, because it would have been terrible as blindness could not be prevented. Even if this is a single experience, we are prompted to say that the diagnosis of WFS1 at the pre-clinical/paucisymptomatic stage may warrant so much caution. Early diagnosis is important to enable proper counselling and to prevent complications, but in the absence of any possible treatment to prevent disease progression, the backlash of diagnosing such a neurodegenerative disorder should be evaluated.

\section{Materials and Methods}

Clinical report. The proband, a female, was the first of two siblings born from healthy nonconsaguineous parents. The brother was healthy. None of the relatives developed diabetes mellitus or any kind of eye disease. She was a full term infant born from caesarion section after abruptio placentae. The neurodevelopmental milestones were regular. Menarche occurred at 10 years old. DM was diagnosed at 11 years of age $(\mathrm{HbA} 1 \mathrm{c}$ $8.5 \%$-DCA2000, no ketosis). Specific autoantibodies against beta-cells were absent. One year later, the insulin requirement was low $(0.29 \mathrm{IU} / \mathrm{kg} /$ day $)$ with optimal blood glucose control (HbA1c constantly < 6.5\%). Specific autoantibodies for DM were newly assyaed and tested negative. Although the family history was not suggestive of DM or dysglycemia, a "de novo mutation" causative of Maturity-Onset Diabetes of the Young (MODY) was suspected in consideration of the evidence that de novo mutations of GCK, HNF-1 $\alpha$ and $H N F-4 \alpha$ may be more frequent in MODY than previously assumed [20]. HNF-4 $4, G C K$, $H N F-1 \alpha, P D X 1, H N F-1 \beta$, and NEUROD1 were sequenced but no pathogenic variants were detected. Consequently, we suggested that further genetic testing could be useful to investigate the etiology of her DM, but at this stage the patient and her parents decided to not undergo further genetic testing to avoid stress and anxiety. During the follow-up, $\mathrm{HbA} 1 \mathrm{c}$ 
values were constantly below 6.5\%, C-peptide levels were in the middle-low normal range and renal function tests were normal. Daily insulin requirement was always low (about $0.2 \mathrm{IU} / \mathrm{kg} /$ day), even during puberty. No severe hypoglycemias or ketoacidosis episodes ever occurred.

At 17.8 years old, she presented a rapid reduction in visual acuity and she was admitted to the Ophtalmologist Unit, where optic subatrophy was diagnosed. On ophthalmic examination, her visual acuity was 2-3/10 (right eye) and 1/10 (left eye). Fundus examination showed pale discs in both eyes. Fluorescein angiography and brain MRI were normal except for partial atrophy of eye nerves. She never complained about hearing impariment and audiogram confirmed a normal hearing function. Due to the copresence of DM and optic subatrophy, Wolfram syndrome was suspected and WFS1 sequencing was requested.

At last clinical evaluation, at 21 years old, the patient was on basal-bolus insulin regimen therapy. Blood glucose control has always been at target $(\mathrm{HbA} 1 \mathrm{c}<7 \%)$ with a very low insulin requirement $(0.18-0.25 \mathrm{IU} / \mathrm{kg} /$ day $)$, weight and height $25-50^{\circ}$ percentiles; last BMI: $21.7 \mathrm{~kg} / \mathrm{m}^{2}$. A recent comprehensive ophthalmic examination described worsening of visual acuity (1/10 in both eyes). She is supported by a psychologist.

DNA extraction. DNA from the proband, parents, and her brother was extracted from whole blood using High Pure PCR Template Preparation Kit (Roche, Mannheim, Germany). This family provided written informed consent to molecular testing and to the full content of this publication.

Sanger Sequencing. Exons and flanking regions of WFS1 were amplified by PCR using previously described primers [21]. Amplicons were purified with exonuclease I and shrimp alkaline phosphatase (ExoSap-IT, USB Corporation, Staufen, Germany) and then sequenced for both sense and antisense strands using an automated fluorescent sequencing method (Big Dye Terminator Kit v1.1, Applied Biosystems, Waltham, MA, USA). The products were separated on an ABI PRISM sequencing apparatus 3730 (Applied Biosystems). All variations were validated by sequencing both DNA strands of three independent PCR products.

SNP-array analysis. High resolution SNP-array analysis was performed on genomic DNA extracted from peripheral blood lymphocytes of the patient, her brother and her parents using the CytoScan HD Array (Thermo Fisher Scientific, Waltham, MA, USA) as previously described [22]. Data analysis was performed using the Chromosome Analysis Suite software version 4.2 (Thermo Fisher Scientific, Waltham, MA, USA). The significance of each of the detected copy number variations (CNVs) was determined by comparing all chromosomal alterations identified in the patient with those collected in an internal database of $\sim 4500$ patients studied by SNP arrays since 2010, and public databases including the Database of Genomic Variants (DGV), DECIPHER, and ClinVar. Base pair positions, information about genomic regions and genes affected by CNVs, and known associated disease have been derived from the University of California Santa Cruz (UCSC) Genome Browser, build GRCh37 (hg19). The clinical significance of each of the rearrangements detected has been assessed following the American College of Medical Genetics (ACMG) guidelines [23].

\section{Conclusions}

In conclusion, we describe the first case of Wolfram syndrome due to uniparental maternal mero-isodisomy UPD4 carrying a novel genetic variant, and presenting with diabetes mellitus at 10 years and optic atrophy at 18 years old. Furthermore, this case calls also for caution in testing patients for wolframin gene defects when they present only diabetes mellitus, as there is currently no effective therapy to prevent disease progression.

Author Contributions: Conceptualization, M.D. and E.P.; methodology, C.A., A.S., O.P., P.P. and M.C.; software, O.P.; validation, P.P. and M.C.; formal analysis, C.A. and A.S.; M.D., F.O. and E.P.; resources, E.P.; data curation, F.C.S.; writing — original draft preparation, M.D., N.R. and O.P.; writing—review and editing, F.C.S.; supervision, E.P.; project administration, M.D. All authors have read and agreed to the published version of the manuscript. 
Funding: This research received no external funding.

Institutional Review Board Statement: The study was conducted according to the guidelines of the Declaration of Helsinki, and approved by the Ethics Committee of Azienda Ospedaliero-Universitaria "Consorziale Policlinico" di Bari (protocol code 6073, approved on 13 November 2019.

Informed Consent Statement: Informed consent was obtained from all subjects involved in the study.

Data Availability Statement: Due to the privacy policy, data are available on request after approval of the patients.

Acknowledgments: The authors thank the patient and her relatives.

Conflicts of Interest: The authors declare no conflict of interest.

\section{References}

1. Tranebjærg, L.; Barrett, T.; Rendtorff, N.D. WFS1 Wolfram Syndrome Spectrum Disorder; Adam, M.P., Ardinger, H.H., Pagon, R.A., Eds.; University of Washington: Seattle, WA, USA, 2009.

2. Inoue, H.; Tanizawa, Y.; Wasson, J.; Behn, P.; Kalidas, K.; Bernal-Mizrachi, E.; Mueckler, M.; Marshall, H.; Donis-Keller, H.; Crock, P.; et al. A gene encoding a trans membrane protein is mutated in patients with diabetes mellitus and optic atrophy. Nat. Gen. 1998, 20, 143-148. [CrossRef] [PubMed]

3. De Heredia, M.L.; Clèries, R.; Nunes, V. Genotypic classification of patients withWolfram syndrome: Insights into the naturalhistory of the disease and correlation with phenotype. Genet. Med. 2013, 15, 497-506. [CrossRef]

4. Rigoli, L.; Bramanti, P.; Di Bella, C.; De Luca, F. Correction: Genetic and clinical aspects of Wolfram syndrome 1, a severe neurodegenerative disease. Pediat. Res. 2018, 84, 787, Erratum for: Pediatr. Res. 2018, 83, 921-929. [CrossRef] [PubMed]

5. Delvecchio, M.; Iacoviello, M.; Pantaleo, A.; Resta, N. Clinical Spectrum Associated with Wolfram Syndrome Type 1 and Type 2: A Review on Genotype-Phenotype Correlations. Int. J. Environ. Res. Public Health 2021, 18, 4796. [CrossRef]

6. Mozzillo, E.; Delvecchio, M.; Carella, M.; Grandone, E.; Palumbo, P.; Salina, A.; Aloi, C.; Buono, P.; Izzo, A.; D’Annunzio, G.; et al. A novel CISD2 intragenic deletion, optic neuropathy and platelet aggregation defect in Wolfram syndrome type 2 . BMC Med. Genet. 2014, 15, 88. [CrossRef]

7. Giuliano, F.; Bannwarth, S.; Monnot, S.; Cano, A.; Chabrol, B.; Vialettes, B.; Delobel, B.; Paquis-Flucklinger, V. Wolfram syndrome in French population: Characterization of novel mutations and polymorphisms in the WFS1 gene. Hum. Mut. 2005, 25, 99-100. [CrossRef] [PubMed]

8. McKinlay, G.R.J.; Amor, D.J. Gardner and Sutherland's Chromosome Abnormalities and Genetic Counseling, 5th ed.; Oxford University Press: Oxford, UK, 2018.

9. Benn, P. Uniparental disomy: Origin, frequency, and clinical significance. Prenat. Diagn. 2021, 41, 564-572. [CrossRef]

10. Spena, S.; Duga, S.; Asselta, R.; Peyvandi, F.; Mahasandana, C.; Malcovati, M.; Tenchini, M.L. Congenital afibrinogenaemia caused byuniparental isodisomy of chromosome 4 containing a novel 15-kb deletion involving fibrinogen Aalpha-chain gene. Eur. J. Hum. Gen. 2004, 12, 891-898. [CrossRef]

11. Cottrell, C.E.; Mendell, J.; Hart-Kothari, M.; Ell, D.; Thrush, D.L.; Astbury, C.; Pastore, M.; Gastier-Foster, J.M.; Pyatt, R.E. Maternal uniparental disomy of chromosome 4 in a patient with limb-girdle muscular dystrophy 2E confirmed by SNP array technology. Clin. Gen. 2012, 81, 578-583. [CrossRef]

12. Ding, Q.; Ouyang, Q.; Xi, X.; Wang, X.; Shen, Y.; Wang, H. Maternal chromosome 4heterodisomy/isodisomy and B $\beta$ chain Trp323X mutation resulting in severe hypodysfibrinogenaemia. Thromb. Haemost. 2012, 108, 654-661. [PubMed]

13. Chen, M.; Hao, H.; Xiong, H.; Cai, Y.; Ma, F.; Shi, C.; Xiao, X.; Li, S. Segmental uniparental disomy of chromosome 4 in a patient with methylmalonic acidemia. Mol. Genet. Genom. Med. 2020, 8, e1063. [CrossRef] [PubMed]

14. Kloth, K.; Vater, I.; Lindschau, R.; Caliebe, A.; Muschol, N.M. Mucopolysaccharidosis type I due to maternal uniparental disomy of chromosome 4 with partial isodisomy of 4p16.3p15.2. Mol. Gen. Metab. Rep. 2020, 25, 100660.

15. Oyarzabal, A.; Martínez-Pardo, M.; Merinero, B.; Navarrete, R.; Desviat, L.R.; Ugarte, M.; Rodríguez-Pombo, P. A novel regulatory defect inthe branched-chain a-keto acid dehydrogenase complex due to a mutation inthe PPM1K gene causes a mild variant phenotype of maple syrup urine disease. Hum. Mut. 2013, 34, 355-362. [CrossRef] [PubMed]

16. Middleton, F.A.; Trauzzi, M.G.; Shrimpton, A.E.; Gentile, K.L.; Morley, C.P.; Medeiros, H.; Pato, M.T.; Pato, C.N. Complete maternal uniparentalisodisomy of chromosome 4 in a subject with major depressive disorderdetected by high density SNP genotyping arrays. Am. J. Med. Gen. 2006, 141, 28-32. [CrossRef]

17. Palumbo, P.; Palumbo, O.; Leone, M.P.; Stallone, R.; Zelante, L.; Carella, M. Maternal uniparental isodisomy (iUPD) of chromosome 4 in a subject with mild intellectual disability and speech delay. Am. J. Med. Gen. 2015, 167, 2219-2222. [CrossRef] [PubMed]

18. Nakka, P.; Pattillo Smith, S.; O’Donnell-Luria, A.H.; McManus, K.F.; Mountain, J.L.; Ramachandran, S.; Sathirapongsasuti, J.F.; 23andMe Research Team. Characterization of Prevalence and Health Consequences of Uniparental Disomy in Four Million Individuals from the General Population. Am. J. Hum. Gen. 2019, 105, 921-932. [CrossRef] [PubMed] 
19. Papadimitriou, D.T.; Manolakos, E.; Bothou, C.; Zoupanos, G.; Papoulidis, I.; Orru, S.; Skarmoutsos, F.; Delides, A.; Bakoula, C.; Papadimitriou, A.; et al. Maternal uniparental disomy of chromosome 4 and homozygous novel mutation in the WFS1 gene in a paediatric patient with Wolfram syndrome. Diabetes Metab. 2015, 41, 433-435. [CrossRef] [PubMed]

20. Stanik, J.; Dusatkova, P.; Cinek, O.; Valentinova, L.; Huckova, M.; Skopkova, M.; Dusatkova, L.; Stanikova, D.; Pura, M.; Klimes, I.; et al. De novo mutations of GCK, HNF1A and HNF4A may be more frequent in MODY than previously assumed. Diabetologia 2014, 57, 480-484. [CrossRef] [PubMed]

21. Colosimo, A.; Guida, V.; Rigoli, L.; Di Bella, C.; De Luca, A.; Briuglia, S.; Stuppia, L.; Salpietro, D.C.; Dallapiccola, B. Molecular detection of novel WFS1 mutations in patients with Wolfram syndrome by a DHPLC-based assay. Hum. Mut. 2003, 21, 622-629. [CrossRef] [PubMed]

22. Palumbo, O.; Palumbo, P.; Di Muro, E.; Cinque, L.; Petracca, A.; Carella, M.; Castori, M. A Private 16q24.2q24.3 Microduplication in a Boy with Intellectual Disability, Speech Delay and Mild Dysmorphic Features. Genes 2020, 11, 707. [CrossRef]

23. Kearney, H.M.; South, S.T.; Wolff, D.J.; Lamb, A.; Hamosh, A.; Rao, K.W.; Working Group of the American College of Medical Genetics. American College of Medical Genetics recommendations for the design and performance expectations for clinical genomic copy number microarrays intended for use in the postnatal setting for detection of constitutional abnormalities. Genet. Med. 2011, 13, 676-679. [CrossRef] [PubMed] 